\title{
Optimization of the Effect of Process Parameters and Study of Quality Characteristics of Osmotic Dehydrated Amla Slices
}

\author{
V.Preethi ${ }^{1 *}$, D.Tiroutchelvame ${ }^{2}$ \\ Department of Food Processing and Engineering, \\ Karunya University, Coimbatore- 641114, Tamilnadu, India
}

\begin{abstract}
The osmotic dehydration technique not only enables the storage of the fruits for a longer period, but also preserves flavour, nutritional characteristics and prevents microbial spoilage. Osmotic Dehydration is done by immersing Vegetable \& Fruits, whether in pieces or whole, in salt or sugar aqueous solution of high osmotic pressure. The amla (Phyllanthus emblica) is an effective source of Vitamin C. The ascorbic acid (Vitamin C) content in the amla fruit is one of the highest contents of all plants. The amla plant has been used in Indian medicine for years. In Ayurvedic medicine, the amla plant is used to treat minor hemorrhage and to help foster cardiovascular health. The study conducted for Amla slices during osmotic dehydration followed by tray drying and the influence of process variables on the quality characteristics. The samples were osmodehydrated at in combination of sugar $\left(25-60^{\circ} \mathrm{Brix}\right)$ and salt $(5 \%, 7.5 \%$ and $10 \%)$ solution at temperature of $30^{\circ} \mathrm{C}$ in $4: 1$ and 8:1 ratio. The treated sample slices were spread on stainless steel trays of the tray dryer and they were dried in tray dryer at temperature $50^{\circ} \mathrm{C}$. The samples were weighed at an interval of 30 minutes. The sample took around 2 to 3 hours depending on the drying temperature to reach equilibrium moisture content. It was observed that the weight reduction, solid gain and weight loss increased with increase of the parameters like time, concentration of the sugar solution and ratio of the solution to sample. The quality parameters such as reducing sugars and ash content were determined.
\end{abstract}

Key Words: Osmotic Dehydration, Amla, Tray Dryer, Weight Reduction, Solid Gain and Weight loss.

\section{Osmotic Dehydration:}

\section{Introduction}

Osmotic dehydration is a process of partial removal of water by soaking foods, mostly fruits and vegetables, in hypertonic solutions (Shi and Maguer 2002). The driving force for the diffusion of water from plant tissue into solution is difference between osmotic pressures of hypertonic solution and plant tissue. The diffusion of water is accompanied by simultaneous counter diffusion of solutes from solution into tissue (Lazarides et al. 1995) Leakage of natural solutes from plant tissue occurs because the cell membranes of plant tissue responsible for osmotic transport is not perfectly selective but this flow is negligible, although it may be important for the organoleptic and nutritional properties of the product (Heng et al. 1990, Mizrahi et al. 2001, Sahoo et al. 2007, Singh et al. 2008). The food which has been osmotically dehydrated can be further processed by freezing, freeze drying vacuum drying and air drying (Nanjundaswamy et al. 1978). Sugar, glucose, fructose, corn syrup and sodium chloride are the common osmotic agents and out of this sodium chloride solution is commonly used for vegetables and sucrose solution for fruits.

\section{Amla:}

Aonla or Indian gooseberry (Emblica officinalis Garten) has wide popularity all over the world because of the medicinal properties. The medicinal preparations are so highly regarded that many scientists have focussed attention on discovering the nutritional and medicinal principles of aonla. The high level of ascorbic acid makes it good as an antiscorbutic, diuretic, laxative and antibiotic [Mehta (1995)]. The fruit is also reported to possess pronounced expectorant, antiviral and cardiotonic activities. It also possesses hypoglycemic activity [Kalra (1988)].

- Osmotic dehydration

\section{Materials And Methods}

- Drying- Tray drying.

- Quality analysis.

- Chemical analysis- Analysis of reducing sugars, Ash content. 
Different Treatment to be performed at each Temperature:

\begin{tabular}{|l|c|}
\hline Temperature $\left({ }^{\circ} \mathbf{c}\right)$ & 30 \\
\hline Ratio of solution to sample & $4: 1,8: 1$ \\
\hline Concentration of osmotic solution & $25-60^{\circ}$ brix \\
\hline Concentration of salt solution $(\%)$ & $5,7.5,10 \%$ \\
\hline Time & 2 hours \\
\hline
\end{tabular}

Fresh amla were purchased locally and thoroughly washed with water to remove adhering soil and other debris. They were blanched and cut into pieces of $1.5 \mathrm{~cm}$ size. Sugar and salt are the osmotic agent was purchased from the local supermarket. The osmotic solution is prepared by mixing the sugar and salt with proper amount of pure water. The osmotic dehydration was conducted in a $500 \mathrm{ml}$ beaker which was placed in a water bath. Sample were cut into small pieces and weighed and then placed into dehydration vessel containing sugar solution of varying concentration $\left(25-60^{\circ} \mathrm{Brix}\right)$ and salt $(5,7.5,15 \%)$. The vessel was placed into the water bath at constant temperature. The solution and sample ratio is 4:1and 8:1. After each half an hour (30 min) the pieces were taken and weighed. In each of the experiment fresh osmotic syrup were used. The treated sample slices were spread on stainless steel trays of the tray dryer and they were dried in tray dryer at temperature $50^{\circ}$ C. The samples were weighed at an interval of 30 minutes. The samples took around 2 to 3 hours depending on the drying temperature to reach equilibrium moisture content. The average moisture and dry matter content of the sample were determined by drying in hot air oven. In order to follow adequately the osmotic dehydration kinetics, individual analysis for each sample were carried out and from these; weight reduction (WR), solid gain (GN) and weight loss (WL) data were obtained, according to the following expression given by Ozen et al. (2002) and Singh et al. (2007)

Weight reduction, $W R=\frac{\mathrm{Wo}_{0}-\mathrm{W}}{\mathrm{W}_{0}} \times 100$

Solid gain, $\mathrm{SG}=\frac{M-M o}{W o} \times 100$

Weight loss, $\mathrm{WL}=\mathrm{WR}+\mathrm{SG}$

Where,

$\mathrm{W}_{0}$ - Initial sample weight $(\mathrm{g})$,

$\mathrm{W}$ - Sample weight after osmotic dehydration (g),

Mo - Initial solid content in the fresh sample (g),

$\mathrm{M}$ - Solid content in the sample after osmotic dehydration (g).

\section{Results And Discussion}

This chapter deals with the experiments conducted on osmotic dehydration of amla. The results obtained are tabulated and discussed in detail.

Concentration of the osmotic agent: sugar $\left({ }^{\circ}\right.$ brix) and salt (5\%)-(4:1):

\begin{tabular}{|l|l|l|l|l|l|l|l|l|}
\begin{tabular}{|l|l|l|l|l|l|} 
Time \\
$(\mathbf{m i n s})$
\end{tabular} & $\mathbf{2 5}$ & $\mathbf{3 0}$ & $\mathbf{3 5}$ & $\mathbf{4 0}$ & $\mathbf{4 5}$ & $\mathbf{5 0}$ & $\mathbf{5 5}$ & $\mathbf{6 0}$ \\
\hline $\mathbf{0}$ & 50 & 50.5 & 50.38 & 49.14 & 50.55 & 49.80 & 49.82 & 49.51 \\
\hline $\mathbf{3 0}$ & 49.58 & 48.63 & 45.45 & 47.68 & 48.93 & 47.63 & 47.23 & 46.32 \\
\hline $\mathbf{6 0}$ & 48.37 & 47.14 & 30.11 & 45.12 & 47.62 & 45.82 & 44.38 & 43.89 \\
\hline $\mathbf{9 0}$ & 47.98 & 46.82 & 37.62 & 43.95 & 45.20 & 43.64 & 42.78 & 43.12 \\
\hline $\mathbf{1 2 0}$ & 47.69 & 39.42 & 36.18 & 41.32 & 42.35 & 41.28 & 41.12 & 41.63 \\
\hline
\end{tabular}

Concentration of the osmotic agent: sugar ( ${ }^{\circ}$ brix) and salt (7.5\%):

\begin{tabular}{|l|l|l|l|l|l|l|l|l|}
\hline $\begin{array}{l}\text { Conc } \\
\text { Time } \\
\text { (mins) }\end{array}$ & $\mathbf{2 5}$ & $\mathbf{3 0}$ & $\mathbf{3 5}$ & $\mathbf{4 0}$ & $\mathbf{4 5}$ & $\mathbf{5 0}$ & $\mathbf{5 5}$ & $\mathbf{6 0}$ \\
\hline $\mathbf{0}$ & 50.07 & 50.73 & 51.57 & 50.0 & 50.14 & 50.56 & 51.16 & 50.53 \\
\hline $\mathbf{3 0}$ & 47.83 & 48.10 & 48.08 & 47.91 & 45.98 & 45.13 & 46.78 & 45.62 \\
\hline $\mathbf{6 0}$ & 45.28 & 45.38 & 45.13 & 44.30 & 41.36 & 40.89 & 41.32 & 40.80 \\
\hline $\mathbf{9 0}$ & 43.61 & 43.12 & 43.86 & 40.18 & 38.12 & 37.63 & 38.49 & 35.31 \\
\hline $\mathbf{1 2 0}$ & 40.99 & 40.41 & 40.4 & 35.80 & 34.36 & 33.11 & 35.42 & 30.48 \\
\hline
\end{tabular}


Optimization of the Effect of process Parameters and Study of Quality characteristics of Osmotic

Concentration of the osmotic agent: sugar $\left({ }^{\circ}\right.$ brix) and salt $(10 \%)$ :

\begin{tabular}{|l|l|l|l|l|l|l|l|l|}
\hline $\begin{array}{l}\text { Conc } \\
\text { Time } \\
\text { (mins) }\end{array}$ & $\mathbf{2 5}$ & $\mathbf{3 0}$ & $\mathbf{3 5}$ & $\mathbf{4 0}$ & $\mathbf{4 5}$ & $\mathbf{5 0}$ & $\mathbf{5 5}$ & $\mathbf{6 0}$ \\
\hline $\mathbf{0}$ & 50.39 & 49.98 & 50.32 & 50.11 & 48.35 & 49.77 & 50.48 & 50.12 \\
\hline $\mathbf{3 0}$ & 47.83 & 45.28 & 47.91 & 46.73 & 44.18 & 45.99 & 45.76 & 44.96 \\
\hline $\mathbf{6 0}$ & 45.40 & 42.73 & 44.32 & 41.28 & 40.92 & 41.18 & 41.69 & 41.38 \\
\hline $\mathbf{9 0}$ & 41.32 & 34.84 & 40.18 & 37.94 & 37.64 & 34.32 & 38.14 & 37.63 \\
\hline $\mathbf{1 2 0}$ & 36.70 & 35.12 & 36.79 & 34.32 & 34.96 & 34.10 & 34.68 & 34.18 \\
\hline
\end{tabular}

WEIGHT REDUCTION-(4:1):

Concentration of the osmotic agent: Salt (5\%) and sugar ( ${ }^{\circ}$ brix):

\begin{tabular}{|l|l|l|l|l|l|l|l|l|}
\hline $\begin{array}{l}\text { Time } \\
\text { (mins) }\end{array}$ & $\mathbf{2 5}$ & $\mathbf{3 0}$ & $\mathbf{3 5}$ & $\mathbf{4 0}$ & $\mathbf{4 5}$ & $\mathbf{5 0}$ & $\mathbf{5 5}$ & $\mathbf{6 0}$ \\
\hline $\mathbf{3 0}$ & 0.84 & 3.70 & 9.78 & 2.97 & 3.20 & 4.35 & 5.19 & 6.44 \\
\hline $\mathbf{6 0}$ & 3.26 & 6.65 & 23.36 & 8.18 & 5.79 & 7.99 & 10.91 & 11.35 \\
\hline $\mathbf{9 0}$ & 4.04 & 7.28 & 25.32 & 10.56 & 10.58 & 12.36 & 14.13 & 12.90 \\
\hline $\mathbf{1 2 0}$ & 4.62 & 21.9 & 28.18 & 15.91 & 16.22 & 17.10 & 17.46 & 15.91 \\
\hline
\end{tabular}

Concentration of the osmotic agent: Salt $(7.5 \%)$ and sugar $\left({ }^{\circ}\right.$ brix):

\begin{tabular}{|l|l|l|l|l|l|l|l|l|}
\hline $\begin{array}{l}\text { Time } \\
(\mathbf{m i n s})\end{array}$ & $\mathbf{2 5}$ & $\mathbf{3 0}$ & $\mathbf{3 5}$ & $\mathbf{4 0}$ & $\mathbf{4 5}$ & $\mathbf{5 0}$ & $\mathbf{5 5}$ & $\mathbf{6 0}$ \\
\hline $\mathbf{3 0}$ & 4.47 & 5.18 & 6.77 & 4.18 & 8.29 & 10.38 & 8.56 & 9.72 \\
\hline $\mathbf{6 0}$ & 9.57 & 10.55 & 12.48 & 11.40 & 17.51 & 18.80 & 19.23 & 19.26 \\
\hline $\mathbf{9 0}$ & 12.90 & 15.0 & 14.95 & 19.64 & 23.97 & 25.27 & 24.76 & 30.12 \\
\hline $\mathbf{1 2 0}$ & 18.13 & 20.34 & 21.65 & 28.4 & 31.47 & 34.25 & 30.77 & 39.68 \\
\hline
\end{tabular}

Concentration of the osmotic agent: Salt (10\%) and sugar ( ${ }^{\circ}$ brix):

\begin{tabular}{|l|l|l|l|l|l|l|l|l|}
\hline $\begin{array}{l}\text { Time } \\
\text { (mins) }\end{array}$ & $\mathbf{2 5}$ & $\mathbf{3 0}$ & $\mathbf{3 5}$ & $\mathbf{4 0}$ & $\mathbf{4 5}$ & $\mathbf{5 0}$ & $\mathbf{5 5}$ & $\mathbf{6 0}$ \\
\hline $\mathbf{3 0}$ & 10.85 & 10.64 & 9.80 & 9.34 & 9.85 & 9.20 & 10.99 & 10.29 \\
\hline $\mathbf{6 0}$ & 3.26 & 8.93 & 23.36 & 8.18 & 5.79 & 7.99 & 10.91 & 11.35 \\
\hline $\mathbf{9 0}$ & 4.04 & 7.28 & 25.32 & 10.56 & 10.58 & 12.36 & 14.13 & 12.90 \\
\hline $\mathbf{1 2 0}$ & 4.62 & 21.9 & 28.18 & 15.91 & 16.22 & 17.10 & 17.46 & 15.91 \\
\hline
\end{tabular}

Ratio 8:1-Concentration of the osmotic agent: sugar ( ${ }^{\circ}$ brix) and salt (5\%):

\begin{tabular}{|l|l|l|l|l|l|l|l|l|}
\begin{tabular}{|l} 
Conc \\
$\begin{array}{l}\text { Cime } \\
\text { (mins) }\end{array}$
\end{tabular} & $\mathbf{2 5}$ & $\mathbf{3 0}$ & $\mathbf{3 5}$ & $\mathbf{4 0}$ & $\mathbf{4 5}$ & $\mathbf{5 0}$ & $\mathbf{5 5}$ & $\mathbf{6 0}$ \\
\hline $\mathbf{0}$ & 50.12 & 50.25 & 50.31 & 50.5 & 50.3 & 50.47 & 50.17 & 50.75 \\
\hline $\mathbf{3 0}$ & 48.36 & 48.13 & 48.23 & 48.10 & 48.13 & 48.73 & 48.69 & 48.96 \\
\hline $\mathbf{6 0}$ & 47.44 & 47.09 & 47.11 & 47.39 & 47.5 & 46.19 & 47.38 & 47.33 \\
\hline $\mathbf{9 0}$ & 46.23 & 45.98 & 46.30 & 46.23 & 46.73 & 44.98 & 45.85 & 46.18 \\
\hline $\mathbf{1 2 0}$ & 45.10 & 44.73 & 45.22 & 45.18 & 45.78 & 43.26 & 44.98 & 45.29 \\
\hline
\end{tabular}


- Concentration of the osmotic agent: sugar ( $\left.{ }^{\circ} \mathrm{brix}\right)$ and salt $(7.5 \%)$ :

\begin{tabular}{|c|c|c|c|c|c|c|c|c|}
\hline $\begin{array}{l}\text { Conc, } \\
\text { Time } \\
\text { (mins }\end{array}$ & 25 & 30 & 35 & 40 & 45 & 50 & 55 & 60 \\
\hline 0 & 49.8 & 50.4 & 50.2 & 50.2 & 50.2 & 49.7 & 50.5 & 50.3 \\
\hline 30 & 48.9 & 49.2 & 49.3 & 49.18 & 49.2 & 48.3 & 48.6 & 48.7 \\
\hline 60 & 47.6 & 48.8 & 48.9 & 48.3 & 48.9 & 47.6 & 46.3 & 47.1 \\
\hline 90 & 46.9 & 47.6 & 48.1 & 47.6 & 47.6 & 46.3 & 45.8 & 46.38 \\
\hline 120 & 45.7 & 46.5 & 47.3 & 46.8 & 45.4 & 45.1 & 44.1 & 44.43 \\
\hline \multicolumn{9}{|c|}{ Concentration of the osmotic agent: sugar $\left({ }^{\circ} \mathrm{brix}\right)$ and salt $(10 \%)$ : } \\
\hline $\begin{array}{l}\text { Conc } \\
\text { Time } \\
\text { (mins) }\end{array}$ & 25 & 30 & 35 & 40 & 45 & 50 & 55 & 60 \\
\hline 0 & 50.10 & 50.63 & 50.23 & 50.61 & 50.3 & 50.32 & 50.12 & 50.71 \\
\hline 30 & 47.62 & 47.88 & 47.30 & 47.97 & 47.22 & 47.11 & 47.67 & 47.38 \\
\hline 60 & 45.14 & 45.36 & 45.68 & 45.73 & 45.34 & 45.48 & 45.40 & 45.60 \\
\hline 90 & 43.29 & 43.99 & 43.29 & 43.98 & 43.16 & 43.55 & 43.79 & 43.99 \\
\hline 120 & 40.46 & 40.12 & 40.91 & 40.79 & 41.01 & 40.98 & 41.12 & 41.69 \\
\hline
\end{tabular}

WEIGHT REDUCTION: 8:1:-

Concentration of the osmotic agent: Salt ( $5 \%$ ) and sugar ( $\mathrm{brix})$ :

\begin{tabular}{|l|l|l|l|l|l|l|l|l|}
\hline $\begin{array}{l}\text { Conc } \\
\text { Timit } \\
\text { (mins) }\end{array}$ & $\mathbf{2 5}$ & $\mathbf{3 0}$ & $\mathbf{3 5}$ & $\mathbf{4 0}$ & $\mathbf{4 5}$ & $\mathbf{5 0}$ & $\mathbf{5 5}$ & 60 \\
\hline $\mathbf{3 0}$ & 3.51 & 4.22 & 5.74 & 3.05 & 4.12 & 4.29 & 4.25 & 2.99 \\
\hline 60 & 5.35 & 6.29 & 9.41 & 6.38 & 5.65 & 5.74 & 5.74 & 5.63 \\
\hline 90 & 7.76 & 8.49 & 11.14 & 8.03 & 7.41 & 8.36 & 8.30 & 7.00 \\
\hline 120 & 10.0 & 10.98 & 12.59 & 9.75 & 8.96 & 10.1 & 10.13 & 8.76 \\
\hline
\end{tabular}

Concentration of the osmotic agent: Salt $(\mathbf{7 . 5 \%})$ and sugar $\left({ }^{\circ} \mathrm{brix}\right)$

\begin{tabular}{|c|c|c|c|c|c|c|c|c|}
\hline $\begin{array}{c}\text { Conc } \\
\text { Tine } \\
\text { (mins) }\end{array}$ & $\mathbf{2 5}$ & $\mathbf{3 0}$ & $\mathbf{3 5}$ & $\mathbf{4 0}$ & $\mathbf{4 5}$ & $\mathbf{5 0}$ & $\mathbf{5 5}$ & $\mathbf{6 0}$ \\
\hline $\mathbf{3 0}$ & 1.81 & 2.38 & 1.79 & 2.03 & 1.99 & 2.82 & 3.76 & 3.18 \\
\hline $\mathbf{6 0}$ & 4.42 & 3.17 & 2.59 & 3.78 & 2.59 & 3.82 & 8.32 & 6.36 \\
\hline $\mathbf{9 0}$ & 5.82 & 5.56 & 4.18 & 5.18 & 5.18 & 6.84 & 9.31 & 7.79 \\
\hline $\mathbf{1 2 0}$ & 8.23 & 7.74 & 7.78 & 6.77 & 9.56 & 9.25 & 12.67 & 11.67 \\
\hline
\end{tabular}

Concentration of the osmotic agent: Salt $(10 \%)$ and sugar $\left({ }^{\circ}\right.$ brix $)$

\begin{tabular}{|c|c|c|c|c|c|c|c|c|}
\hline $\begin{array}{c}\text { Conc } \\
\text { Time } \\
\text { (mins) }\end{array}$ & $\mathbf{2 5}$ & $\mathbf{3 0}$ & $\mathbf{3 5}$ & $\mathbf{4 0}$ & $\mathbf{4 5}$ & $\mathbf{5 0}$ & $\mathbf{5 5}$ & $\mathbf{6 0}$ \\
\hline $\mathbf{3 0}$ & 4.95 & 5.43 & 5.83 & 5.22 & 6.12 & 6.38 & 4.89 & 6.57 \\
\hline $\mathbf{6 0}$ & 9.90 & 10.41 & 9.06 & 9.64 & 9.86 & 9.62 & 9.42 & 10.1 \\
\hline $\mathbf{9 0}$ & 13.59 & 13.11 & 13.82 & 13.10 & 14.19 & 13.45 & 12.63 & 13.25 \\
\hline $\mathbf{1 2 0}$ & 19.24 & 20.79 & 18.55 & 19.40 & 18.47 & 18.56 & 17.96 & 17.78 \\
\hline
\end{tabular}

The weight reduction of Amla increased from $(0.84-10.0 \%),(3.7-10.98 \%),(2.78-12.59 \%),(2.97-9.75 \%),(3.2-$ $8.96 \%),(4.35-10.1 \%),(5.19-10.13 \%),(6.44-8.76 \%)$ for all the treatments with increase in temperature $30^{\circ} \mathrm{C}$ with increase in sugar and salt $(5 \%)$ concentration from $25-60^{\circ}$ brix and solution to sample ratio from $4: 1$ to 8:1.The weight reduction of Amla increased from (4.47-8.23\%), (5.18-7.74\%), (6.77-7.78\%), (4.18-6.77\%), $(8.29-9.56 \%),(9.25-10.38 \%),(8.56-12.67 \%),(9.72-11.67 \%)$ for all the treatments with increase in temperature $30^{\circ} \mathrm{C}$ with increase in sugar and salt $(7.5 \%)$ concentration from $25-60^{\circ}$ brix and solution to sample ratio from $4: 1$ to 8:1. The weight reduction of Amla increased from (5.08-19.24\%), (9.40-20.79\%), (4.79-18.55\%), (6.75$19.40 \%),(8.62-18.47 \%),(7.59-18.56 \%),(8.69-17.96 \%),(9.15-17.78 \%)$ for all the treatments with increase in temperature $30^{\circ} \mathrm{C}$ with increase in sugar and salt $(10 \%)$ concentration from $25-60^{\circ}$ brix and solution to sample ratio from $4: 1$ to $8: 1$. 


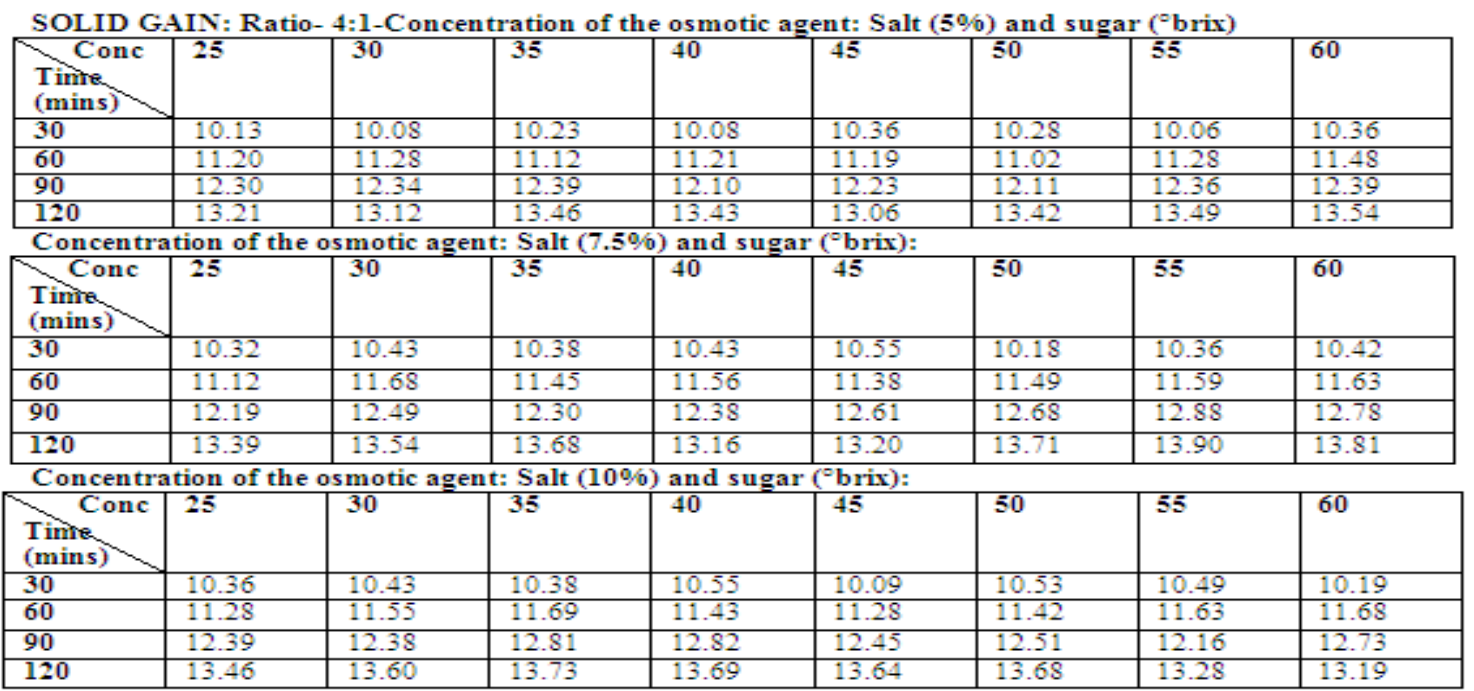

$\mathrm{M}=$ solid content in sample after osmotic dehydration.

$\mathrm{Mo}=$ solid content in fresh sample, $0.84 \mathrm{~g}$,

Wo $=$ initial sample weight, $50 \mathrm{~g}$

Concentration of the osmotic agent: Salt (5\%) and sugar ( $\left.{ }^{\circ} \mathrm{brix}\right)$ :

\begin{tabular}{|l|l|l|l|l|l|l|l|l|}
\hline $\begin{array}{l}\text { Conc } \\
\text { Time } \\
\text { (mins) }\end{array}$ & $\mathbf{2 5}$ & $\mathbf{3 0}$ & $\mathbf{3 5}$ & $\mathbf{4 0}$ & $\mathbf{4 5}$ & $\mathbf{5 0}$ & $\mathbf{5 5}$ & $\mathbf{6 0}$ \\
\hline $\mathbf{3 0}$ & 18.58 & 18.48 & 18.78 & 18.48 & 19.04 & 18.88 & 18.44 & 19.04 \\
\hline 60 & 20.72 & 20.88 & 20.56 & 20.74 & 20.7 & 20.36 & 20.88 & 21.28 \\
\hline 90 & 22.92 & 23.0 & 23.10 & 22.52 & 22.78 & 22.54 & 23.04 & 23.10 \\
\hline $1 \mathbf{2 0}$ & 24.74 & 24.56 & 25.24 & 25.18 & 24.44 & 25.16 & 25.16 & 25.40 \\
\hline
\end{tabular}

Concentration of the osmotic agent: Salt $(7.5 \%)$ and sugar ( $\left.{ }^{\circ} \mathrm{brix}\right)$ :

\begin{tabular}{|l|l|l|l|l|l|l|l|l|}
\hline $\begin{array}{l}\text { Conc } \\
\text { Timis } \\
\text { (mins) }\end{array}$ & $\mathbf{2 5}$ & $\mathbf{3 0}$ & $\mathbf{3 5}$ & $\mathbf{4 0}$ & $\mathbf{4 5}$ & $\mathbf{5 0}$ & $\mathbf{5 5}$ & $\mathbf{6 0}$ \\
\hline $\mathbf{3 0}$ & 18.96 & 19.18 & 19.08 & 19.18 & 19.42 & 18.68 & 19.04 & 19.16 \\
\hline 60 & 20.56 & 21.68 & 21.22 & 21.44 & 21.08 & 21.30 & 21.50 & 21.58 \\
\hline 90 & 22.70 & 23.30 & 20.92 & 23.08 & 23.54 & 23.68 & 24.08 & 23.88 \\
\hline 120 & 25.10 & 25.40 & 25.68 & 24.64 & 24.72 & 25.74 & 26.12 & 25.94 \\
\hline
\end{tabular}

Concentration of the osmotic agent: Salt $(10 \%)$ and sugar $\left({ }^{\circ} \mathrm{brix}\right)$ :

\begin{tabular}{|l|l|l|l|l|l|l|l|l|}
\hline $\begin{array}{l}\text { Conc } \\
\text { Timivs } \\
\text { (mins) }\end{array}$ & $\mathbf{2 5}$ & $\mathbf{3 0}$ & $\mathbf{3 5}$ & $\mathbf{4 0}$ & $\mathbf{4 5}$ & $\mathbf{5 0}$ & $\mathbf{5 5}$ & $\mathbf{6 0}$ \\
\hline $\mathbf{3 0}$ & 19.04 & 19.18 & 19.08 & 19.42 & 18.50 & 19.38 & 19.30 & 18.70 \\
\hline 60 & 20.88 & 21.42 & 21.70 & 21.18 & 20.88 & 21.16 & 21.58 & 21.68 \\
\hline 90 & 23.10 & 23.08 & 23.94 & 23.96 & 23.22 & 23.34 & 22.64 & 23.78 \\
\hline 120 & 25.24 & 25.52 & 25.78 & 25.70 & 25.60 & 25.68 & 24.88 & 24.70 \\
\hline
\end{tabular}




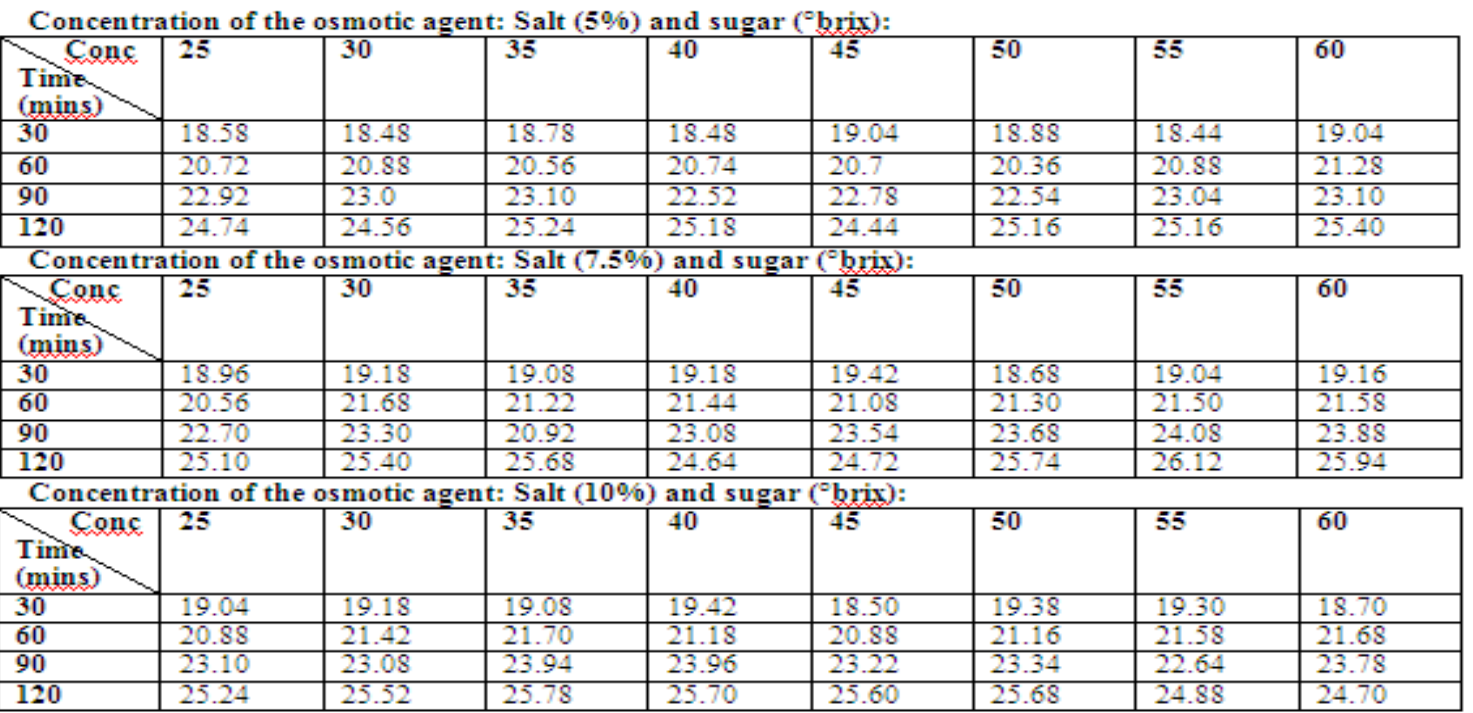

RATIO 8:1-Concentration of the osmotic agent: Salt (5\%) and sugar ( ${ }^{\circ}$ brix):

\begin{tabular}{|l|l|l|l|l|l|l|l|l|}
\begin{tabular}{|l} 
Conc \\
Time \\
(mins)
\end{tabular} & $\mathbf{2 5}$ & $\mathbf{3 0}$ & $\mathbf{3 5}$ & $\mathbf{4 0}$ & $\mathbf{4 5}$ & $\mathbf{5 0}$ & $\mathbf{5 5}$ & $\mathbf{6 0}$ \\
\hline $\mathbf{3 0}$ & 10.42 & 10.35 & 10.48 & 10.59 & 10.62 & 10.53 & 10.48 & 10.51 \\
\hline $\mathbf{6 0}$ & 11.38 & 11.40 & 11.54 & 11.63 & 11.52 & 11.64 & 11.51 & 11.39 \\
\hline $\mathbf{9 0}$ & 12,54 & 12.39 & 12.47 & 12.48 & 12.61 & 12.49 & 12.63 & 12.45 \\
\hline $\mathbf{1 2 0}$ & 13.70 & 13.61 & 13.58 & 13.36 & 13.73 & 13.58 & 13.56 & 13.62 \\
\hline
\end{tabular}

Concentration of the osmotic agent: Salt (7.5\%) and sugar ( ${ }^{\circ}$ brix):

\begin{tabular}{|l|l|l|l|l|l|l|l|l|}
\hline $\begin{array}{l}\text { Conc } \\
\text { Time } \\
\text { (mins) }\end{array}$ & $\mathbf{2 5}$ & $\mathbf{3 0}$ & $\mathbf{3 5}$ & $\mathbf{4 0}$ & $\mathbf{4 5}$ & $\mathbf{5 0}$ & $\mathbf{5 5}$ & $\mathbf{6 0}$ \\
\hline $\mathbf{3 0}$ & 10.38 & 10.49 & 10.27 & 10.39 & 10.45 & 10.51 & 10.43 & 10.55 \\
\hline $\mathbf{6 0}$ & 11.46 & 11.34 & 11.31 & 11.45 & 11.36 & 11.49 & 11.55 & 11.38 \\
\hline $\mathbf{9 0}$ & 12.27 & 12.45 & 12.52 & 12.62 & 12.51 & 12.62 & 12.34 & 12.24 \\
\hline $\mathbf{1 2 0}$ & 13.53 & 13.36 & 13.49 & 13.54 & 13.63 & 13.78 & 13.28 & 13.46 \\
\hline
\end{tabular}

Concentration of the osmotic agent: Salt (10\%) and sugar ( ${ }^{\circ}$ brix):

\begin{tabular}{|l|l|l|l|l|l|l|l|l|}
\begin{tabular}{|}
$\begin{array}{c}\text { Conc } \\
\text { Time } \\
(\mathbf{m i n s})\end{array}$ \\
$\mathbf{3 0}$
\end{tabular} & $\mathbf{2 5}$ & $\mathbf{3 0}$ & $\mathbf{3 5}$ & $\mathbf{4 0}$ & $\mathbf{4 5}$ & $\mathbf{5 0}$ & $\mathbf{5 5}$ & $\mathbf{6 0}$ \\
\hline $\mathbf{6 0}$ & 11.67 & 10.37 & 10.61 & 10.68 & 10.45 & 10.51 & 10.43 & 10.55 \\
\hline $\mathbf{9 0}$ & 12.36 & 12.61 & 12.55 & 12.36 & 12.59 & 12.62 & 12.34 & 12.24 \\
\hline $\mathbf{1 2 0}$ & 13.61 & 13.57 & 13.68 & 13.45 & 13.33 & 13.78 & 13.28 & 13.46 \\
\hline
\end{tabular}

$\mathrm{M}=$ solid content in sample after osmotic dehydration.

Mo $=$ solid content in fresh sample, $0.84 \mathrm{~g}$,

Wo $=$ initial sample weight, $50 \mathrm{~g}$

Concentration of the osmotic agent: Salt (5\%) and sugar $\left({ }^{\circ}\right.$ brix $)$ :

\begin{tabular}{|l|l|l|l|l|l|l|l|l|}
\hline $\begin{array}{l}\text { Gonc } \\
\text { Time } \\
\text { (mins) }\end{array}$ & $\mathbf{2 5}$ & $\mathbf{3 0}$ & $\mathbf{3 5}$ & $\mathbf{4 0}$ & $\mathbf{4 5}$ & $\mathbf{5 0}$ & $\mathbf{5 5}$ & $\mathbf{6 0}$ \\
\hline $\mathbf{3 0}$ & 19.16 & 19.02 & 19.28 & 19.50 & 19.56 & 19.38 & 19.28 & 19.34 \\
\hline $\mathbf{6 0}$ & 21.08 & 21.12 & 21.42 & 21.58 & 21.38 & 21.60 & 21.36 & 21.10 \\
\hline $\mathbf{9 0}$ & 23.40 & 23.10 & 23.26 & 23.28 & 23.54 & 23.30 & 23.58 & 23.22 \\
\hline $\mathbf{1 2 0}$ & 25.72 & 25.54 & 25.48 & 25.04 & 25.78 & 25.48 & 25.44 & 25.56 \\
\hline
\end{tabular}


Concentration of the osmotic agent: Salt $(\mathbf{7 . 5 \%})$ and sugar $\left({ }^{\circ}\right.$ brix):

\begin{tabular}{|l|l|l|l|l|l|l|l|l|}
\hline $\begin{array}{l}\text { Gonc } \\
\text { Time } \\
\text { (mins) }\end{array}$ & $\mathbf{2 5}$ & $\mathbf{3 0}$ & $\mathbf{3 5}$ & $\mathbf{4 0}$ & $\mathbf{4 5}$ & $\mathbf{5 0}$ & $\mathbf{5 5}$ & $\mathbf{6 0}$ \\
\hline $\mathbf{3 0}$ & 19.08 & 19.30 & 18.86 & 19.10 & 19.22 & 19.34 & 19.18 & 19.42 \\
\hline $\mathbf{6 0}$ & 21.24 & 21.00 & 20.94 & 21.22 & 21.04 & 21.30 & 21.44 & 21.08 \\
\hline $\mathbf{9 0}$ & 22.86 & 23.22 & 23.30 & 23.56 & 23.28 & 23.50 & 23.00 & 22.80 \\
\hline $\mathbf{1 2 0}$ & 13.58 & 25.04 & 25.30 & 25.40 & 25.58 & 25.88 & 24.88 & 25.24 \\
\hline
\end{tabular}

Concentration of the osmotic agent: Salt $(\mathbf{1 0 \%})$ and sugar $\left({ }^{\circ} \mathrm{brix}\right)$ :

\begin{tabular}{|l|l|l|l|l|l|l|l|l|}
\begin{tabular}{|l} 
Time \\
(mins)
\end{tabular} & $\mathbf{2 5}$ & $\mathbf{3 0}$ & $\mathbf{3 5}$ & $\mathbf{4 0}$ & $\mathbf{4 5}$ & $\mathbf{5 0}$ & $\mathbf{5 5}$ & $\mathbf{6 0}$ \\
\hline $\mathbf{3 0}$ & 19.36 & 19.06 & 19.54 & 19.68 & 19.22 & 19.38 & 19.60 & 19.10 \\
\hline $\mathbf{6 0}$ & 21.66 & 21.22 & 19.16 & 19.14 & 21.08 & 21.24 & 21.36 & 21.22 \\
\hline $\mathbf{9 0}$ & 23.04 & 23.54 & 23.42 & 23.04 & 23.50 & 22.82 & 23.30 & 23.28 \\
\hline $\mathbf{1 2 0}$ & 25.54 & 25.46 & 25.68 & 25.22 & 24.98 & 25.08 & 25.56 & 25.04 \\
\hline
\end{tabular}

The solid gain of Amla increased from (18.58-25.72\%), (18.48-25.54\%), (18.78-25.48\%), (18.48-25.04\%), $(19.04-25.78 \%),(18.88-25.48 \%),(18.44-25.44 \%),(19.04-25.56 \%)$ for all the treatments with increase in temperature $30^{\circ} \mathrm{C}$ with increase in sugar and salt $(5 \%)$ concentration from $25-60^{\circ}$ brix and solution to sample ratio from 4:1 to 8:1. The solid gain of Amla increased from (18.96-23.58\%), (19.18-25.04\%), (19.08-25.30\%), (19.18-25.40\%), (19.42-25.58\%), (18.68-25.88\%), (19.04-24.88\%), (19.16-25.24\%) for all the treatments with increase in temperature from $30^{\circ} \mathrm{C}$ with increase in sugar and salt $(7.5 \%)$ concentration from $25-60^{\circ}$ brix and solution to sample ratio from 4:1 to 8:1. The solid gain of Amla increased from (19.04-25.54\%), (19.18$25.46 \%),(19.08-25.68 \%),(19.42-25.22 \%),(18.50-24.98 \%),(19.38-25.08 \%),(19.30-25.56 \%),(18.70-25.04 \%)$ for all the treatments with increase in temperature from $30^{\circ} \mathrm{C}$ with increase in sugar and salt $(10 \%)$ concentration from $25-60^{\circ}$ brix and solution to sample ratio from $4: 1$ to $8: 1$.

WEIGHT LOSS: Ratio 4:1-Concentration of the osmotic agent: Salt (5\%) and sugar:

\begin{tabular}{|l|l|l|l|l|l|l|l|l|}
\begin{tabular}{|l}
$\begin{array}{l}\text { Conc } \\
\text { Time } \\
\text { (mins) }\end{array}$ \\
$\mathbf{3 0}$
\end{tabular} & $\mathbf{2 5}$ & $\mathbf{3 0}$ & $\mathbf{3 5}$ & $\mathbf{4 0}$ & $\mathbf{4 5}$ & $\mathbf{5 0}$ & $\mathbf{5 5}$ & $\mathbf{6 0}$ \\
\hline $\mathbf{6 0}$ & 23.42 & 22.18 & 28.56 & 21.45 & 22.24 & 17.23 & 23.63 & 25.48 \\
\hline $\mathbf{9 0}$ & 26.96 & 27.53 & 42.92 & 28.88 & 26.49 & 28.35 & 31.79 & 32.63 \\
\hline $\mathbf{1 2 0}$ & 29.36 & 46.28 & 48.42 & 33.08 & 33.36 & 34.9 & 37.17 & 36.0 \\
\hline
\end{tabular}

Concentration of the osmotic agent: Salt (7.5\%) and sugar ( ${ }^{\circ}$ brix):

\begin{tabular}{|l|l|l|l|l|l|l|l|l|}
\hline $\begin{array}{l}\text { Conc } \\
\text { Time } \\
\text { (mins) }\end{array}$ & $\mathbf{2 5}$ & $\mathbf{3 0}$ & $\mathbf{3 5}$ & $\mathbf{4 0}$ & $\mathbf{4 5}$ & $\mathbf{5 0}$ & $\mathbf{5 5}$ & $\mathbf{6 0}$ \\
\hline $\mathbf{3 0}$ & 23.43 & 24.36 & 25.85 & 23.36 & 27.71 & 29.06 & 27.6 & 28.88 \\
\hline $\mathbf{6 0}$ & 30.13 & 32.23 & 33.7 & 32.84 & 38.59 & 40.1 & 40.73 & 40.84 \\
\hline $\mathbf{9 0}$ & 35.6 & 38.3 & 35.87 & 42.72 & 47.51 & 48.95 & 48.84 & 54.0 \\
\hline $\mathbf{1 2 0}$ & 43.23 & 45.74 & 47.43 & 53.04 & 56.19 & 59.99 & 56.89 & 65.62 \\
\hline
\end{tabular}

Concentration of the osmotic agent: Salt $(10 \%)$ and sugar $\left({ }^{\circ}\right.$ brix):

\begin{tabular}{|l|l|l|l|l|l|l|l|l|}
\begin{tabular}{|l}
$\begin{array}{l}\text { Conc } \\
\text { Time } \\
\text { (mins) }\end{array}$ \\
$\mathbf{3 0}$
\end{tabular} & $\mathbf{2 5}$ & $\mathbf{3 0}$ & $\mathbf{3 5}$ & $\mathbf{4 0}$ & $\mathbf{4 5}$ & $\mathbf{5 0}$ & $\mathbf{5 5}$ & $\mathbf{6 0}$ \\
\hline $\mathbf{6 0}$ & 20.06 & 28.88 & 24.36 & 20.5 & 20.01 & 19.78 & 23.01 & 23.7 \\
\hline $\mathbf{9 0}$ & 26.31 & 40.84 & 32.23 & 23.29 & 23.31 & 24.72 & 26.93 & 30.93 \\
\hline $\mathbf{1 2 0}$ & 30.68 & 65.62 & 45.3 & 26.66 & 26.58 & 26.76 & 33.61 & 39.37 \\
\hline
\end{tabular}


Optimization of the Effect of process Parameters and Study of Quality characteristics of Osmotic

Ratio 8:1-Concentration of the osmotic agent: Salt (5\%) and sugar ( ${ }^{\circ}$ brix):

\begin{tabular}{|l|l|l|l|l|l|l|l|l|}
\begin{tabular}{|l} 
Time \\
Conc \\
(mins)
\end{tabular} & $\mathbf{2 5}$ & $\mathbf{3 0}$ & $\mathbf{3 5}$ & $\mathbf{4 0}$ & $\mathbf{4 5}$ & $\mathbf{5 0}$ & $\mathbf{5 5}$ & $\mathbf{6 0}$ \\
\hline $\mathbf{3 0}$ & 22.67 & 23.24 & 25.02 & 22.55 & 23.68 & 23.67 & 23.53 & 22.33 \\
\hline $\mathbf{6 0}$ & 26.43 & 27.41 & 30.83 & 27.96 & 27.03 & 27.34 & 27.1 & 26.73 \\
\hline $\mathbf{9 0}$ & 31.16 & 31.59 & 34.4 & 31.31 & 30.95 & 31.66 & 31.88 & 30.22 \\
\hline $\mathbf{1 2 0}$ & 35.72 & 36.52 & 38.07 & 34.79 & 34.74 & 35.58 & 35.57 & 34.32 \\
\hline
\end{tabular}

Concentration of the osmotic agent: Salt $(\mathbf{7 . 5 \%})$ and sugar $\left({ }^{\circ}\right.$ brix):

\begin{tabular}{|l|l|l|l|l|l|l|l|l|}
\begin{tabular}{|l|l|l|l|l|} 
Conc \\
Time \\
(mins)
\end{tabular} & $\mathbf{2 5}$ & $\mathbf{3 0}$ & $\mathbf{3 5}$ & $\mathbf{4 0}$ & $\mathbf{4 5}$ & $\mathbf{5 0}$ & $\mathbf{5 5}$ & $\mathbf{6 0}$ \\
\hline $\mathbf{3 0}$ & 20.89 & 21.68 & 20.65 & 21.13 & 21.21 & 22.16 & 22.94 & 22.6 \\
\hline $\mathbf{6 0}$ & 25.66 & 24.17 & 23.53 & 25.00 & 23.63 & 25.12 & 29.76 & 27.44 \\
\hline $\mathbf{9 0}$ & 21.68 & 28.78 & 27.48 & 28.74 & 28.46 & 30.34 & 32.31 & 30.59 \\
\hline $\mathbf{1 2 0}$ & 28.81 & 32.78 & 31.08 & 32.17 & 35.14 & 35.13 & 37.55 & 36.91 \\
\hline
\end{tabular}

Concentration of the osmotic agent: Salt (10\%) and sugar $\left({ }^{\circ}\right.$ brix):

\begin{tabular}{|l|l|l|l|l|l|l|l|l|}
\begin{tabular}{|l} 
Conc \\
$\begin{array}{c}\text { Cime } \\
\text { (mins) }\end{array}$
\end{tabular} & $\mathbf{2 5}$ & $\mathbf{3 0}$ & $\mathbf{3 5}$ & $\mathbf{4 0}$ & $\mathbf{4 5}$ & $\mathbf{5 0}$ & $\mathbf{5 5}$ & $\mathbf{6 0}$ \\
\hline $\mathbf{3 0}$ & 24.31 & 24.49 & 25.37 & 24.9 & 25.34 & 25.76 & 24.49 & 25.67 \\
\hline $\mathbf{6 0}$ & 31.56 & 31.63 & 28.22 & 28.78 & 30.94 & 30.86 & 30.78 & 31.32 \\
\hline $\mathbf{9 0}$ & 36.63 & 36.65 & 37.24 & 36.14 & 37.69 & 36.27 & 35.93 & 36.53 \\
\hline $\mathbf{1 2 0}$ & 44.78 & 46.25 & 44.23 & 44.62 & 43.45 & 43.64 & 43.52 & 42.82 \\
\hline
\end{tabular}

The weight loss of Amla increased from (19.42-35.72\%), (22.18-36.52\%), (28.56-38.07\%), (21.45-34.79\%), (22.24-34.74\%), (17.23-35.58\%), (23.63-35.57\%), (25.48-34.32\%) for all the treatments with increase in temperature $30^{\circ} \mathrm{C}$ with increase in sugar and salt $(5 \%)$ concentration from $25-60^{\circ}$ brix and solution to sample ratio from 4:1 to 8:1. The weight loss of Amla increased from (23.43-28.81\%), (24.36-32.78\%), (25.85$31.08 \%),(23.36-32.17 \%),(27.71-35.14 \%),(29.06-35.13 \%),(27.6-37.55 \%),(28.88-36.91 \%)$, for all the treatments with increase in temperature $30^{\circ} \mathrm{C}$ with increase in sugar and salt $(7.5 \%)$ concentration from 25$60^{\circ}$ brix and solution to sample ratio from 4:1 to 8:1. The weight loss of Amla increased from (20.06-44.78\%), (28.88-46.25\%), (24.36-44.23\%), (20.5-44.62\%), (20.01-43.45\%), (19.78-43.64\%), (23.01-43.52\%), (23.7$42.82 \%$ ), for all the treatments with increase in temperature $30^{\circ} \mathrm{C}$ with increase in sugar and salt $(10 \%)$ concentration from $25-60^{\circ}$ brix and solution to sample ratio from $4: 1$ to $8: 1$.

Chemical analysis - Ash content for the osmotic dehydration of Amla: [Ranganna S (1986)]

\begin{tabular}{|l|l|l|}
\hline S.No & Concentration of the sample & Ash content(g) \\
\hline 1 & Control (raw Amla sample) & 0.3 \\
\hline 2 & Concentration of salt $(15 \%), 30^{\circ} \mathrm{C}, 4: 1$ & 0.14 \\
\hline 3 & Concentration of sugar $\left(45^{\circ}\right.$ brix $), 30^{\circ} \mathrm{C}, 4: 1$ & 0.23 \\
\hline 4 & Concentration of sugar $\left(50^{\circ}\right.$ brix $), \operatorname{salt}(10 \%), 30^{\circ} \mathrm{C}, 4: 1$ & 0.18 \\
\hline
\end{tabular}

From the above table, it was observed that the ash content for the concentration of salt $(15 \%)$ is $0.14 \mathrm{~g}$, Concentration of sugar $\left(45^{\circ} \mathrm{brix}\right)$ is $0.23 \mathrm{~g}$, Concentration of $\operatorname{sugar}\left(50^{\circ} \mathrm{brix}\right)$ and salt $(10 \%)$ is $0.18 \mathrm{~g}$ was analyzed for the raw Amla sample to the osmotic dehydrated of Amla at $30^{\circ} \mathrm{C}, 4: 1$ ratio. When compared to other concentrations of sugar and salt, concentration of sugar $\left(45^{\circ} \mathrm{brix}\right), 30^{\circ} \mathrm{C}, 4: 1$ is high.

Chemical analysis - Analysis of Reducing Sugars for the osmotic dehydration of Amla:[Lane, J. H. and Eynon, L. (1923)]

\begin{tabular}{|l|l|l|}
\hline S.No & Concentration of the sample & Reducing Sugar \\
\hline 1 & Concentration of sugar $\left(60^{\circ}\right.$ brix $), 30^{\circ} \mathrm{C}, 4: 1$ & 22.3 \\
\hline 2 & Concentration of sugar $\left(40^{\circ}\right.$ brix $), 30^{\circ} \mathrm{C}, 4: 1$ & 21.5 \\
\hline 3 & Concentration of sugar $\left(50^{\circ} \mathrm{brix}\right), 30^{\circ} \mathrm{C}, 8: 1$ & 22.1 \\
\hline 4 & Concentration of $\operatorname{sugar}\left(60^{\circ} \mathrm{brix}\right)$, salt $(5 \%) 30^{\circ} \mathrm{C}, 8: 1$ & 23.5 \\
\hline
\end{tabular}


From the above table, the Reducing sugar for the Concentration of sugar $\left(60^{\circ} \mathrm{brix}\right), 30^{\circ} \mathrm{C}, 4: 1$ is 22.3 , Concentration of sugar $\left(40^{\circ}\right.$ brix $), 30^{\circ} \mathrm{C}, 4: 1$ is 21.5 , Concentration of sugar $\left(50^{\circ}\right.$ brix $), 30^{\circ} \mathrm{C}, 8: 1$ is 22.1 , Concentration of sugar $\left(60^{\circ}\right.$ brix $)$, salt $(5 \%) 30^{\circ} \mathrm{C}, 8: 1$ is 23.5 was analyzed for the osmotically dehydrated Amla. [AOAC (2000) Official methods of analysis].

\section{Conclusion}

It was concluded that the study conducted for Amla slices during osmotic dehydration followed by tray drying and the influence of process variables on the quality characteristics. The samples were osmo dehydrated in combination of sugar $\left(25-60^{\circ} \mathrm{Brix}\right)$ and salt $(5 \%, 7.5 \%, 10 \%)$ solution at temperature $30^{\circ} \mathrm{C}$ in $4: 1$ and $8: 1$ ratio. It was observed that the weight reduction, solid gain and water loss increased with increased in the parameters like concentration of sugar solution, salt solution and ratio of solution to sample and time

\section{References}

[1]. AOAC (2000) Official methods of analysis (984.25). 17th edn, Association of Official Analytical Chemists, Maryland

[2]. Heng W, Guilbert S, Cuq JL (1990) Osmotic dehydration of papaya: influence of process variables on the product quality. Sci Aliments 10:832-848.

[3]. Kalra CL (1988) The chemistry and technology of Amla (Phyllanthus emblica): A resume. Indian Food Packer 38(4): 67-82.

[4]. Lane, J. H. and Eynon, L. (1923). Volumetric determination of reducing sugars by means of Fehling's solution, with methylene blue as internal indicator. IS1 XXV : 143-149.

[5]. Lazarides HN, Katsanidis E, Nickolaides A (1995) Mass transfer kinetics during osmotic pre concentration aiming at minimal solid uptake. J Food Eng 35:151.

[6]. Mehta S (1995) Evaluation of Different Cultivars of Aonla (Emblica officinalis Garten) for Processing. MSc Thesis, CCS HAU, Hisar.

[7]. Mizrahi S, Eicheler S, Raman O (2001) Osmotic dehydration in gel systems. J Food Eng 49:87-96.

[8]. Nanjundaswamy AM, Radhakrishnaiah SG, Balachandran C, Saroja S, Murthy Reddy KBS (1978) Studies on development of new categories of dehydrated products from indigenous fruits. Indian Food Packer 32(1):91-99.

[9]. Ozen BF, Dock LL, Ozdemir M, Floros JD (2002) Processing factors affecting the osmotic dehydration of diced green peppers. Int J Food Sci Technol 37:497-502.

[10]. Ranganna S (1986) Handbook of Analysis and Quality Control for Fruit \& Vegetable Products. 2nd edn. Tata McGraw Hill Publishing Co. Ltd., New Delhi.

[11]. Sahoo PK, Mitra J, Chakraborty S (2007) Kinetics of apparent mass diffusivities on osmotic dehydration of carrots. J Food Sci Technol 44:26-28.

[12]. Shi J, Maguer M le (2002) Osmotic dehydration of foods: Mass transfer and modeling aspects. Food Rev Int 18:305-336.

[13]. Singh B, Kumar A, Gupta AK (2007) Study of mass transfer kinetics and effective diffusivity during osmotic dehydration of carrot cubes. J Food Eng 79:471-480.

[14]. Singh C, Sharma HK, Sarkar BC (2008) Optimization of process conditions during osmotic dehydration of fresh pineapple. J Food Sci Technol 45:305-311. 